NASA Technical Memorandum 81818

\title{
NASA-TM-81818 19800015192
}

MINIMUM MASS SIZING OF A

LARGE LOW-ASPECT RATIO AIRFRAME

FOR FLUTTER-FREE PERFORMANCE

William H. Greene

and

Jaroslaw Sobieszczanski-Sobieski

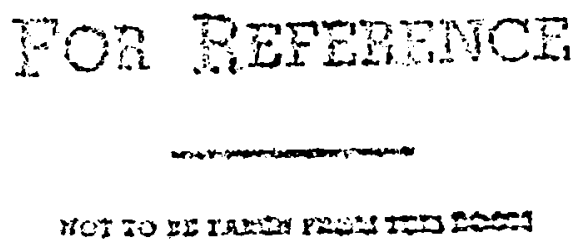

May 1980

\section{N/SA}

National Aeronautics and

Space Administration

Langley Research Center

Hampton, Virginia 23665 


\section{SUMMARY}

A procedure for sizing an airframe for flutter-free performance is demonstrated on a large, flexible supersonic transport aircraft. The procedure is based on using a two level reduced basis or modal technique for reducing the computational cost of performing the repetitive flutter analyses. The supersonic transport aircraft exhibits complex dynamic behavior, has a well-known flutter problem and requires a large finite element model to predict the vibratory and flutter response.

Flutter-free designs are produced with small mass increases relative to the wing structural weight and aircraft payload. In view of the ability of the resizing procedure to handle this supersonic transport configuration, it seems likely that the method could be used for many other aircraft.

\section{INTRODUCTION}

The design of aircraft to meet flutter criteria has received more attention in recent years as larger, more flexible, higher performance aircraft are being developed. The difficulties of intuitively modifying a given design to alleviate a flutter problem are widely recognized, and thus considerable research has been carried out to develop automated design procedures considering flutter constraints. A review of the work in this area to 1974 is presented in reference 1.

The difficulties in developing efficient techniques for automated design stem from the complexity of the flutter phenomenon and the computational cost associated with predicting this behavior. A's modifications are made to the structure, the flutter behavior may change in a complex and highly nonlinear way. Also, if the constraints against flutter are formulated simply as a requirement that the lowest flutter speed exceed some required value, the values of the constraints may be discontinuous functions of the structural changes.

Solutions to the problems of the nonlinear, discontinuous behavior of the flutter constraints and the high computational cost have been proposed. Reference 2 formulates the flutter constraints considering all critical points on all 
flutter modes. This insures that the flutter constraints will not have discontinuous values during the design process. However, formulating the constraints in this way may increase the cost of the repetitive flutter calculations. To alleviate the high cost of repetitive calculations, an approximate analys is technique utilizing a reduced basis method is proposed in reference 3 . This technique provides a means for rapidly recalculating the flutter constraints during the design process. The continuous constraint formulation and this approximate analysis method are coupled in the flutter resizing system described in reference 3. The performance of the system was demonstrated in reference 3 by application to a relatively simple problem--that of a clipped delta wing. However, these techniques have not been applied to a problem with complex dynamic behavior which requires a large mathematical model to predict flutter.

An example of such a complex flutter design problem is a very large and flexible supersonic cruise transport with an arrow-wing. Reference 4 points to the design of this class of aircraft to meet flutter requirements as a major problem.

The purpose of the present study is to apply the flutter redesign techniques described in reference 3 to this difficult problem of an arrow-wing transport. Specifically, the objective is to take an airframe sized for strength considerations only and produce a flutter-free design while minimizing the mass added to the airframe. The geometrical and structural complexities of this airframe suggest that a method shown successful for this case is likely to be general enough to apply to many otiner airframe configurations.

This paper contains a description of the analytical model of the supersonic transport airframe. The resizing method is described in detail along with its performance in the application to the supersonic transport. Finally, the implementation of this flutter design procedure within a general engineering analysis package which includes finite element structural analysis, data management facilities, and a flexible control language is discussed. 


\section{SYMBOLS}
$[\mathrm{A}],[\tilde{\mathrm{A}}],[\overline{\mathrm{A}}]$
matrix of unsteady aerodynamic influence coefficients and generalized aerodynamic force matrices of orders $m_{1}$ and $m_{2}$, respectively
flutter constraint
damping
$g_{\max }$
value of the assumed structural damping
$g_{\text {ref }}$
a reference value of damping
$[K],[\tilde{K}],[\bar{K}]$
structural stiffness matrix and matrices of generalized stiffness coefficients of orders $m_{1}$ and $m_{2}$, respectively
M
Mach number
$[M],[\tilde{M}],[\bar{M}]$
structural mass matrix and matrices of generalized mass coefficients of orders $m_{1}$ and $m_{2}$, respectively
number of degrees of freedom in the finite element model
$\mathrm{m}_{1}$
number of vibration modes used in reducing the order of the approximate vibration problem
$\mathrm{m}_{2}$
number of approximate vibration modes used in reducing the order of the flutter eigenvalue problem
n the number of design variables dynamic pressure, equation (9) $m_{1} \times m_{2}$ matrix of approximate, generalized vibration mode shapes for the modified structure
$r$ factor in equation (10) $m_{2} \times m_{2}$ matrix of generalized flutter mode shapes
flutter speed 


$\begin{array}{ll}v_{\text {req }} & \text { the required flutter speed } \\ v_{i} & \text { value of the } i \text { th design variable } \\ w & \text { mass penalty added to meet the flutter requirements } \\ {[\Delta]} & m_{0} \times m_{0} \text { matrix of natural vibration modes } \\ {[\delta]} & m_{1} \times m_{1} \text { matrix of generalized vibration mode shapes } \\ s 2 & \text { diagonal matrix of vibration frequencies } \\ \omega & \text { diagonal matrix of flutter frequencies } \\ {[\phi]} & \begin{array}{l}m_{0} \times m_{1} \text { matrix of vibration mode shapes generated at the } \\ \text { beginning of each cycle }\end{array}\end{array}$

BASEL INE ANALYTICAL MODEL

The finite element model of the arrow-wing supersonic transport configuration used in this study is described in reference 5 . Its basic characteristics are given in this section. The flutter resizing procedure is applied to an airframe that has already been sized to satisfy static load requirements. The static loading cases and the procedure for sizing the airframe for strength criteria are described in references 5 and 6.

The essential information for the baseline configuration, shown in the table of figure 1, includes the take-off gross weight, operational empty weight, and payload. The wing is built of corrugated web spars and ribs with caps supporting honeycomb sandwich covers. Conventional stringer-skin-frame construction is used in the fuselage. Titanium is used throughout the primary structure.

\section{Finite Element Model}

The finite element model representation of this configuration and the types of construction are shown in figure 2. In the finite element model, the covers are simulated by membrane elements, spar and rib webs by shear panels, and caps by rod elements. Beam elements are used to represent the engines, the engine mounts, and the supports for leading- and trailing-edge devices. Plate 
elements are used to model the vertical fins and horizontal stabilizers. Nonstructural components are represented by appropriate lumped and distributed masses. For computational economy, the fuselage model is simplified to a rectangular cross section thin-walled box with a bending stiffness and mass distribution equivalent to the fuselage. This simplification is consistent with the study's emphasis on the primary wing structure; only elements in the wing structure are resized. The resulting half airplane finite element model has 746 grid points, 2141 degrees of freedom, and 2369 finite elements.

\section{Sizing for Strength Requirements}

From the multitude of loading cases considered in the design of airframes, three cases were selected for use in the strength sizing. The cruise case defines the jig shape and accounts approximately for fatigue, the maneuver case generates the largest wing root bending moment, and the taxi loads expose the wing lower surface covers to compression. Cross sectional dimensions established in the process of strength sizing include thicknesses of the face sheets of the wing cover sandwich panels and thicknesses of the rib and spar webs, while the spar and rib caps remained constant. A typical distribution of the wing cover thicknesses obtained in this manner and which constitute a starting design for the subsequent flutter resizing are shown in figure 3 .

\section{Dynamic Behavior}

Because of its large size and flexible wings the airframe exhibits a complex vibratory behavior. This behavior is shown in deformed plots of the wing planform for the four lowest vibration modes (fig. 4). Although the flexible wing tip contributes most heavily to the modal deformation, there is considerable coupling with the inboard delta-like portion of the wing and with the fuselage, and a pronounced chordwise bending deformation which distinguishes the arrowwing's behavior from that of a conventional high-aspect-ratio wing.

Figure 5 shows the results of the flutter analysis for the airframe after a strength sizing has been performed. It can be seen that the aircraft is severely flutter deficient relative to the required flutter speeds at both Mach .6 and .9. Previous studies have shown that these are the critical Mach numbers 
for this aircraft and both are considered in the flutter resizing procedure. The altitudes at which the flutter analyses are performed for these two Mach numbers were selected to produce the required dynamic pressures to satisfy the necessary margins on flutter speed relative to the flight envelope. They are -2499 and 4267 meters, respectively, for the two Mach numbers. The horizontal axes in the $\mathrm{V}-\mathrm{g}$ plots of figure 5 are located at $g=.02$ which is the value of structural damping assumed for the airframe. It is important to note that three different flutter modes are close to the required flutter speed at both Mach .6 and .9. Thus, it is important for this application that the flutter resizing procedure be able to handle multiple modes.

\section{DESCRIPTION OF THE FLUTTER RESIZING METHOD}

The resizing method discussed here is essentially that of reference 3 with several modifications. The principal ingredients of the method are an approximate flutter analysis using reduced basis techniques, the use of a general purpose optimizer based on nonlinear mathematical programming, a continuous flutter constraint formulation, and a strategy for periodically recalculating the natural vibration modes by solving the global eigenvalue problem. These topics are discussed in the following sections. A summary of the steps in the resizing procedure is also presented.

\section{Reducing the Size of the Flutter Analysis Problem}

For an airframe represented by a complex structural model, such as the supersonic transport, a single flutter analysis can be computationally expensive. This flutter analysis is often performed using a modal method where the lowest natural vibration modes for the airframe are used as generalized coordinates to reduce the dimensionality of the problem. The natural vibration modes are used since a relatively small number frequently provides an accurate representation of the structure's dynamic behavior. The two major steps in the modal flutter

analysis are: (1) calculation of a set of natural vibration modes for the airframe and (2) solution of the complex flutter eigenvalue problem. The first step is particularly expensive if there is a large number of degrees of freedom in the structural model. The second step is expensive if the number of modes used as generalized coordinates is large. 
The cost of both of these steps must be considered in an automated design procedure with flutter constraints. Each time the airframe is modified, the flutter analysis is performed. Thus it is not computationally practical to either calculate the natural vibration modes from the global eigenvalue problem or to employ a large number of modes in the flutter eigenvalue problem in each flutter analysis.

One approach to the problem of reducing the cost is to use natural vibration modes for the unmodified structure as generalized coordinates in solving the flutter equations for the modified structure. The drawbacks of this approach are that either a large number of modes are required in the flutter solution or the natural modes must be regenerated fairly frequently by solving the global eigenvalue problem.

To alleviate these drawbacks a method was proposed in reference 3 which is based on using an approximate analysis to calculate the set of vibration modes for use in the flutter calculations. This approximate analysis is based on a modal approach (similar to that used in the solution of the flutter eigenvalue problem) where the vibration modes for the modified structure are represented by a linear combination of the natural modes from a previous solution of the global, finite element equations. The participation factors in this linear combination are determined by solving a small vibration problem of the order of the number modes in the linear combination. This number of modes used as approximation functions is larger than the number of modes used in reducing the flutter eigenvalue problem but is much smaller than the number of degrees of freedom in in the finite element mode1. A much larger number of generalized degrees of freedom can be used in the approximate vibration problem compared with the flutter eigenvalue problem because the vibration problem is solved only once for each modified structure. This approximate technique drastically cuts the cost of the vibration analysis and provides a good set of approximate modes for the flutter analysis. When the structure changes significantly from the design when the global vibration problem was previously solved, the accuracy of the approximate modes may be unacceptable and a new set of modes must be calculated from the global problem; however, this is required only a few times for a complete design run. 
The first step in the approximate flutter analys is is the solution of the global vibration problem

$$
[K][\Delta]=\Omega^{2}[M][\Delta]
$$

for the set of $m_{1}$ lowest modes and frequencies. The matrices $[K],[M]$, and $[\Delta]$ are of the order of the total number of degrees of freedom in the finite element model. The set of lowest $m_{1}$ vibration modes is denoted $[\phi]$. Note that the value of $m_{1}$ is significantly larger than the number of modes to be used in solving the flutter eigenvalue problem, $m_{2}$. Employing the transformation

$$
[\Delta]=[\phi][\delta]
$$

the generalized stiffness, mass, and aerodynamic force matrices

$$
\begin{aligned}
& {[\tilde{K}]=[\phi]^{\top}[K][\phi]} \\
& {[\tilde{M}]=[\phi]^{\top}[M][\phi]} \\
& {[\tilde{A}]=[\phi]^{\top}[A][\phi]}
\end{aligned}
$$

and derivatives of the stiffness and mass matrices with respect to the design variables

$$
\begin{aligned}
& {\left[\tilde{K}_{j}\right]=[\phi]^{\top}\left[\partial K / \partial v_{j}\right][\phi]} \\
& {\left[\tilde{M}_{i}\right]=[\phi]^{\top}\left[\partial M / \partial v_{j}\right][\phi]}
\end{aligned}
$$

are formed. By virtue of equation (1), $[K]$ and $[M]$ are diagonal. The matrices of aerodynamic influence coefficients, [A] in equation (4), can be calculated for the different Mach numbers and reduced frequencies using any appropriate linear, unsteady aerodynamics theory. The generalized derivative matrices, $\left[\tilde{K}_{i}\right]$ and $\left[\tilde{M}_{j}\right]$ are formed so that generalized stiffness and mass matrices for the modified structure can be formed exactly and quickly by Taylor expansion. The generalized matrices can be formed exactly by Taylor expansion because the membrane finite elements used are linear functions of the design variables. For given values of the design variables representing a modified structure designated by $j$, the generalized matrices are 


$$
\begin{aligned}
& {\left[\tilde{K}_{j}\right]=[\tilde{K}]+\sum_{i=1}^{n}\left[\tilde{K}_{j}\right] v_{i}} \\
& {\left[\tilde{M}_{j}\right]=[\tilde{M}]+\sum_{i=1}^{n}\left[\tilde{M}_{j}\right] v_{i}}
\end{aligned}
$$

These generalized matrices form the reduced, approximate vibration problem

$$
\left[\tilde{K}_{j}\right][\delta]=\Omega_{j}^{2}\left[\tilde{M}_{j}\right][\delta]
$$

Since this eigenvalue problem is only of order $m_{1}$, the lowest $m_{2}$ modes can be calculated with very small cost. This set of $m_{2}$, lowest, approximate vibration modes is denoted [q]. Employing the transformation

$$
[\delta]=[\mathrm{q}][\mathrm{u}]
$$

the generalized stiffness mass, and aerodynamic force matrices of order $m_{2}$

$$
\begin{aligned}
& {\left[\bar{K}_{j}\right]=[q]^{\top}\left[\tilde{K}_{j}\right][q]} \\
& {\left[\bar{M}_{j}\right]=[q]^{\top}\left[\tilde{M}_{j}\right][q]} \\
& {\left[\bar{A}_{j}\right]=[q]^{\top}[\tilde{A}][q]}
\end{aligned}
$$

are computed for the modified structure. The complex flutter eigenvalue problem

$$
\left[\left[\omega^{2}\left[\bar{M}_{j}\right]+(1+i g)\left(\bar{K}_{j}\right]+q\left[\bar{A}_{j}\right]\right][u]=[0]\right.
$$

which is of order $m_{2}$ can be solved to give values of velocity and damping for the reduced frequencies and the flutter modes used in calculating the constraints. In equation (9) $w$ is the flutter frequency, $g$ is a damping parameter, and $q$ is the dynamic presure.

The operations indicated in equations (5) through (9) are performed for every modified structure in the design process. However, these operations are relatively inexpensive. The operations indicated in equations (1) through (4) are relatively costly but are performed only a few times during a design run. Additional comments on the approximate vibration analysis are presented in Appendix A. 


\section{Formulation of the Flutter Constraints}

The flutter constraint formulations of references 2 and 3 are designed to prevent any flutter modes from violating the flutter requirements at any point in the design process. As the airframe is resized, it is highly possible that flutter modes which were not initially critical will become critical. These flutter modes initially may have crossed the $g=g_{\max }$ axis at speeds higher than the required speed or may not have crossed at all (this is the so-called "hump" mode). The constraint formulation of references 2 and 3 considers both cases.

In this study a modification was made to the constraint formulation of reference 3 because of the different optimization algorithm used. A general purpose optimizer, denoted CONMIN (ref. 7), based on a feasible directions method was employed rather than the penalty function method of reference 3 . In order to permit deletion of unimportant constraints during the design process and satisfy the requirements of CONMIN regarding the ordering of constraints passed to it, a cumulative constraint formulation was used.

This cumulative constraint is defined from the sketch of a typical $\mathrm{V}-\mathrm{g}$ diagram.

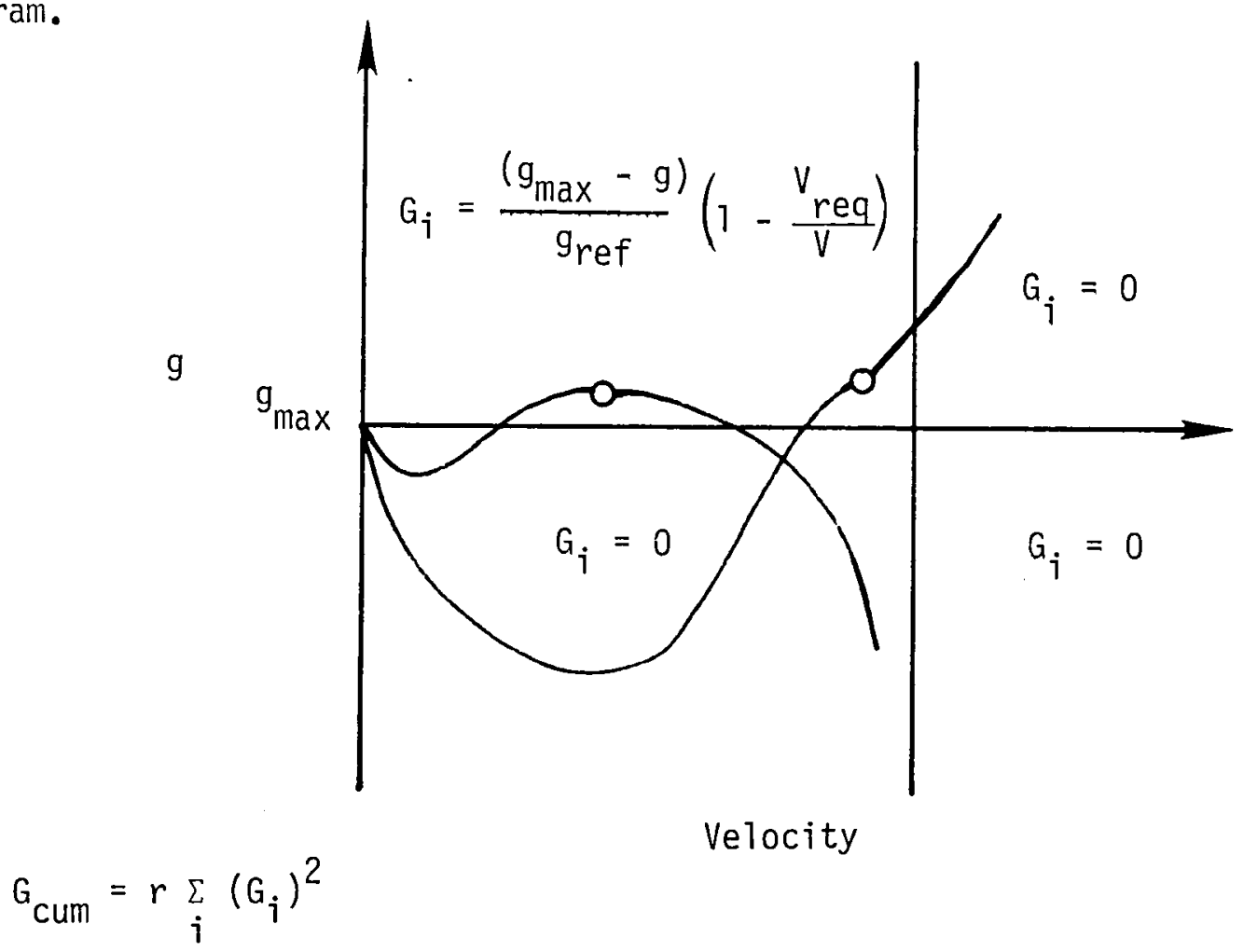


The values of $G_{j}$ in the four regions of the $V$-g diagram are shown in the sketch; a value of $G_{i}>0$ indicates violation of the constraint. The parameter $g_{\text {ref }}$ is a reference value of damping and $r$ is a constant multiplier discussed below. The circles indicate typical $\mathrm{V}-\mathrm{g}$ points at values of the reduced frequency that contribute to the constraint. Constraints for other Mach numbers or altitudes being considered are also summed in equation (10). Thus the complete flutter behavior of the aircraft is represented by the single constraint, equation (10).

Equation (10) is similar in form to an external penalty function formulation where the penalty is zero in the feasible domain. When the external penalty function is used in conjunction with unconstrained minimization techniques, care must be taken in selection of $r$ to avoid numerical difficulties. When equation (10) is used with the feasible directions algorithm, however, there appears to be no problem selecting a very large value of $r$ to force the values of $G_{i}$ toward zero. A typical value of $r$ is 1000 which is used throughout the constrained minimization.

Strategy for Updating the Vibration Modes

As the design changes significantly from that when the global vibration problem was previously solved, the approximate vibration analysis may become unacceptably inaccurate. The global problem is then re-solved for this new, modified structure. The difficulty is selecting the appropriate criterion for returning to the global problem.

In reference 3 , changes in the lowest natural vibration frequency are used in a criterion to determine when to recalculate the complete set of vibration modes. When the approximate frequency for the modified structure differs from the previously calculated "exact" frequency, a new set of exact vibration modes and frequencies are calculated. An advantage of this criterion is that it attempts to directly measure differences in the vibration behavior between the structure for which exact modes were calculated and the modified structure. A disadvantage is that the first vibration mode and frequency may not be one of the dominant ones in the flutter analysis; this is the case for certain flutter conditions of the supersonic transport model. Other, more rigorous strategies 
involving all frequencies and/or modes are possible but these have not been investigated and would be more costly computationally.

In this application, the indirect approach of controlling the accuracy of the approximate analysis by specifying "move limits" on the design variables is used. This approach was used successfully in reference 8 and has the advantage of being easy to implement. Since a single design variable controls a number of individual elements and since the design variables are panel thickness additions, the move limits are related to the thickest panel in each design variable region. The upper and lower values for a particular design variable are a certain percentage above and below the thickness of this specified panel. A minimum gage requirement of zero is also placed on the lower value which specifies that no reductions of the thicknesses in the strength-sized design are allowed. These values are supplied to the optimizer which performs a complete minimization based on these bounds. The process of solving the global vibration problem, prescribing move limits, and performing a complete minimization of the mass is called a cycle.

As the design procedure moves from cycle to cycle, the move limit percentage is reduced by a prescribed factor. The approach is to start with relatively liberal move limits and then tighten the range of the approximate analysis near the final design. Both the initial percentage and the reduction factor are determined by experimentation with a particular problem.

\section{Summary of Steps in the Flutter Resizing Procedure}

The resizing procedure is outlined in the flow chart in figure 6 . The operations in each of the steps of the flow chart are as follows:

1. Select the group of structural parameters controlled by each design variable. In the application reported here, the design procedure is being applied to a structure already sized for strength considerations and the design variables are thickness additions to cover panels on the upper and lower surfaces of the wing. To reduce the number of the design variables considered, the thickness additions to a number of adjacent finite elements are controlled by a single design variable. 
2. Compute the derivatives of the mass with respect to the design variables

$$
w_{i}=\partial w / \partial v_{i}, \quad i=1, n
$$

Since the design variables are thicknesses, the $w_{j}$ are independent of the values of the design variables and are computed only once.

3. Form the mode independent aerodynamic force coefficients at the different Mach numbers being considered for a specific set of reduced frequencies for the important lifting surfaces (both the wing and tail are considered in this study). These coefficients depend only on Mach number, reduced frequency, and surface geometry which is not changed during redesign. Thus, these coefficients are calculated only once. The aerodynamic calculations are based on the kernel function method for subsonic flow and the computer implementation described in reference 8. These aerodynamic coefficients are denoted $[A]$.

4. Form the global finite element stiffness and mass matrices based on the current values of the design variables.

5. From the global equations, calculate a set of $m_{7}$ natural vibration modes and frequencies. The number of modes, $m_{1}$, should be significantly larger than the number of modes finally used in the flutter calculations. This set of $m_{1}$ modes is denoted $[\phi]$.

6. Form the generalized stiffness and mass matrices of order $m_{7}$ (eq. 3) and their derivatives with respect to the design variables (eq. 4).

7. Form the matrix of generalized aerodynamic forces using the set of $m_{1}$ natural vibration modes. The modal deflections at the structural node points are interpolated using spline functions to

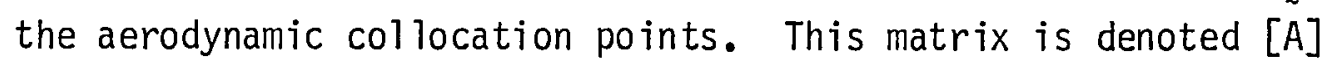
(eq. 3). 
8. Based on the current values of the design variables and the move limit factor, determine the upper and lower move limits for each design variable.

9. For given values of the design variables, $v_{i}$, compute the mass penalty and the generalized stiffness and mass matrices by Taylor expansion (eq. 5). The mass penalty is

$$
w_{j}=\sum_{i=1}^{n} w_{i} v_{i}
$$

10. Solve the reduced eigenvalue problem of order $m_{1}$ (eq. 6) for a set of $m_{2}$ vibration modes and frequencies $\left(m_{2}<m_{1}\right)$. These are approximations to the lowest $m_{2}$ vibration modes for the modified structure.

11. Compute generalized stiffness, mass, and aerodynamic matrices of order $m_{2}$ (eq. 8). Note that $[\bar{K}]$ and $[\bar{M}]$ are always diagonal and this fact can be exploited in the flutter eigensolution method.

12. Solve the complex flutter eigenvalue problem (eq. 9) at the Mach numbers and altitudes being considered. Constraints and possibly derivatives of the constraints with respect to the design variables (depending on the request by the optimizer) are calculated for any critical points on the $V$-g diagram.

13. Execute the optimizer which determines a new set of design variables and requests an analysis for this design or indicates that a minimum mass has been found. If a minimum has not been found, go back to step 9 and continue; otherwise, go to Step 14.

14. If the maximum number of cycles has not been reached, go to step 4 to update the $m_{1}$ vibration mode set by solving the global equations. At this time the factor specifying the move limits on the design variables is reduced by the specified percentage. The maximum number of cycles is predetermined based on the character of the problem and the cost of solving the global eigenvalue problem. The maximum number of cycles is typicaliy from 3 to 10. 
15. Perform a calculation of vibration modes and flutter speeds for the final design to insure that the flutter requirements are met.

The implementation of the above procedure in a general purpose engineering anatysis system is discussed in Appendix B.

\section{APPLICATION AND RESULTS}

A number of numerical studies have been performed to demonstrate the application of the previously described flutter resizing method to a supersonic transport configuration. Mass penalties, values of the design variables, and V-g diagrams for the final designs are presented to characterize the flutterfree aircraft. Design iteration histories for both the mass penalty and key design variables are presented to show the behavior of the method.

\section{Determination of the Numbers of Modes}

The two key parameters of the resizing method which must be selected are: (1) $m_{2}$, the number of modes used in the $V-g$ flutter analysis and (2) $m_{1}$, the number of modes used in the approximate vibration analysis. A convergence study was performed using the strength-sized aircraft to determine how the number of modes used affects the calculated flutter speeds. The results of this study for both Mach .6 and .9 are shown in figure 7. After considering the computational cost of calculating the flutter constraints during the design process, the value of $m_{2}=12$ was selected. As can be seen from figure 7, flutter analysis with 12 modes results in a flutter speed that is approximately $5 \%$ high at both Mach numbers. To counteract this unconservative analysis a modification factor, $f_{v}$, is used to increase the required flutter speed, $v_{\text {req }}$, used in calculating the flutter constraints. Even though the error between the 12 mode flutter solution and the converged solution is not constant during the design process, the initial error provides a guideline for selecting $f_{v}$.

The constant $m_{1}$ should be considerably larger than $m_{2}$ but small enough so that the calculation of $m_{1}$ vibration modes from the global equations is computationally practical. Another factor which limits the size of $m_{1}$ is the cost of computing the generalized matrices (operations indicated in equations 3 
and 4). Although $m_{1}$ is also the order of the reduced free vibration problem (equation 6), the cost of solving this eigenproblem is negligible relative to the above operations. Based on these considerations $m_{1}$ was selected to be 24 . This number of modes is also used in the flutter analysis of the final design which is performed only once. For this application, the computational cost reductions are achieved specifically by reducing the dimensionality of the problem from 2141 degrees of freedom in the global equations, to 24 degrees of freedom in the reduced vibration problem, to 12 degrees of freedom in the flutter eigenvalue problem.

\section{Definition of the Design Variables}

The flutter resizing procedure begins from a design sized for strength considerations. This strength-sized design was produced with the thickness of each individual finite element (both membrane wing skins and shear panel spar and rib webs) controlled by a design variable. This large number of design variables is not practical in the flutter resizing procedure. Only the wing cover skin thicknesses are changed during flutter resizing and a number of finite elements are controlled by a single design variable.

Each design variable represents a thickness addition to all elements in that particular region. Thus the material added to the strength-sized wing can be considered as a series of "patches" made to prevent flutter.

Two different sets of design variable regions were considered in this study; both sets are shown in figure 8. In set I, each design variable controls skin thicknesses on both the upper and lower wing surfaces. In set II, the skins on the lower wing surface are controlled by design variables different from those on the upper surfaces; the layouts of the regions on upper and lower surfaces, as can be seen in figure 8 , are identical. In design variable set I there are 12 variables or regions, in set II there are 24 variables. The purpose of the two sets of design variables originated from a consideration of the initial, strength-sized design. The upper and lower wing cover panels tend to be designed by different load cases; for the upper panels, the maneuver case is the most important and for the lower panels, the taxi load case is the most severe. The result is that after sizing for strength considerations, the upper 
and lower skin thickness distributions are asymmetric. If the optimizer had the freedom to increase the skin thicknesses of upper and lower panels independently, a more symmetric design might be achieved. The intuition that a more efficient design for meeting the flutter requirements would result from making the wing skins more symmetric is based on the higher overall bending stiffness achieved.

\section{Number of Design Cycles}

Since each cycle requires re-solving the global eigenvalue problem, it is important to keep the number of cycles small. It is possible for a very large and complex model, that calculation of vibration modes and frequencies more than once might be impractical. To assess the potential of the method for handling this case, two of the four design studies were performed using only a single cycle. The modification factor, $f_{v}$, was used to account for any discrepancies between the approximate analysis in the design process and the final analysis. Some numerical experimentation was required to select $f_{v}$ but after the appropriate value was determined, correlation between the approximate flutter speeds and those from the final analysis was excellent.

Design studies using a total of five resizing cycles and controlled move limits were also carried out. Results from these studies are compared with results from a single cycle in subsequent sections.

Final Resized, Flutter-Free Designs

The results of four design cases are presented in this section. The four cases are:

A - resizing with the 12 design variable set (I) and only a single cycle.

$B$ - resizing with the 24 design variable set (II) and only a single cycle.

C - resizing with the 12 design variable set and five cycles.

D - resizing with the 24 design variable set and five cycles. 
The values of the design variables and the mass penalties for each of the four designs are shown in figure 9. The mass penalty represents the amount of material added to the total airframe to prevent flutter. In all four designs the upper and lower skins in the tip portion of the wing have been thickened considerably. Material added in this area can act both as a "balancing mass" and to stiffen this flexible tip. Since this portion of the wing is nearly symmetric after strength-sizing, nearly equal additions were made to upper and lower skins. Large thickness additions have also been made between the tip and delta portions of the wing, the region controlled by design variables 5 and 17. In this region the upper and lower wing skins are highly asymmetric after strength-sizing. For design cases $B$ and $D$, considerably more material is added to the lower skin to produce a more symmetric design. The wing region controlled by design variables 10 and 22 is consistently thickened in all flutter-resized designs even though it is a considerable distance from the more flexible area of the wing. However, the wing carry-through structure is located near this region. A structural modification of this type might easily be overlooked using intuitive techniques for resizing the wing to prevent flutter.

The mass penalties associated with the four designs are as expected. Design case $A$ has by far the largest mass penalty but it was produced with the crudest analysis (a single design cycle) and considered only 12 design variables. Improving the design procedure by including more refined analyses (cases $C$ and $D$ ) or additional design variables (cases $B$ and $D$ ) lowers the mass penalty. The lightest flutter-free design, case $D$, has a mass penalty which is only $7 \%$ of the wing structural mass and $6.4 \%$ of the aircraft's payload. This illustrates the modest size of the mass penalties added by the design procedure.

Figure 10 shows a set of skin thickness contours for the upper and lower wing skins for the final flutter-resized design (case D). Comparison with figure 3 shows the relatively subtle changes made to the skin thickness distribution.

Table I compares the values of the lowest natural vibration frequencies of the strenth-sized airframe with those of the flutter-resized airframe. Even though 20\% increases in the flutter speeds have resulted from resizing, the changes in the vibration frequencies are fairly small. The change in the first natural frequency, which is used as a mode update criteria in reference 3 , is only $1 \%$. 
Figure 11 shows the V-g diagrams for the flutter-resized design (case D) at Mach .6 and .9. As mentioned previously, two flutter modes are important at both Mach numbers for the strength-sized design. The resizing procedure has driven the design in a direction such that at both Mach numbers, both modes are nearly critical. This is perfectly consistent with the engineer's intuitive philosophy that simultaneous failure in multiple modes often accompanies a minimum mass design. It also clearly demonstrates that the resizing procedure can effectively account for multiple critical flutter modes.

To gain additional insight into the behavior of the flutter-resizing process, design histories of the mass penalty and selected design variables are shown in figure 12. The mass penalty and the design variables are plotted versus the iteration number where an iteration is defined in the feasible directions algorithm as a single linear minimization in a specified direction in the design space. Histories of the mass penalties are presented for all four design cases; histories of selected design variables are presented for design case D. The flat portions of the design variable histories indicate where a particular design variable reaches a move limit (upper bound) in a particular cycle. With only one design cycle, the design variables have no move limits and the optimizer drives the design quickly to a high mass in order to produce an acceptable (flutter-free) design. It then quickly reduces the mass to achieve the minimum mass design for this case. With five design cycles and move limits on the design variables, the optimizer is not allowed to make these very large changes in the structure early in the design process. For these designs (cases $C$ and $D$ ) the mass penalty is increased to only a moderate value and then slowly decreased to the minimum value. 


\section{CONCLUDING REMARKS}

A procedure for sizing an airframe for flutter-free performance has been demonstrated on a large, flexible supersonic transport aircraft. The procedure is substantially that of reference 3 and is based on using reduced basis or modal techniques for reducing the computational cost of performing flutter analyses. This supersonic transport aircraft exhibits complex dynamic behavior, has a wellknown flutter problem and requires a large finite element model to predict vibratory and flutter response; thus, resizing this airframe to alleviate flutter is a very difficult problem.

From the numerical design studies made it is possible to draw the following conclusions:

1. Flutter-free designs of the aircraft with small mass penalties have been produced. For case $D$, the mass penalty is only $7 \%$ of the wing structural mass and $6 \%$ of the aircraft's payload.

2. The two level modal analysis drastically reduces the cost of the repetitive flutter calculations and makes resizing using the complex finite element model feasible.

3. Execution of the resizing procedure with only a single solution of the finite element vibration problem and using a modification factor on the required flutter speed produced a design with a moderately small mass penalty. For case B, the mass penalty is only $9 \%$ of the wing structural mass and $8 \%$ of the payload.

4. The procedure can effectively handle multiple flutter modes as evidenced by the final designs having two modes simultaneously critical.

5. In view of the ability of the resizing procedure to consider the complex dynamic behavior of this airframe and produce a flutter free design with only a small mass penalty, it seems likely that the method could be used for many other aircraft. 
APPENDIX A

\section{COMMENTS ON THE METHODS FOR APPROXIMATE AND REFINED VIBRATION ANALYSIS}

In this appendix the approximate vibration reanalys is technique used in the procedure described herein is shown to be a subset of the technique used for solving the global finite element eigenvalue problem. Features of the global finite element eigensolution technique which are important when the method is used for reanalysis are described.

The technique used for solving the global eigenvalue problem is known as simultaneous inverse iteration or the subspace iteration method (ref. 10). In this method the desired eigenvectors are represented as a linear combination of shapes

$$
[X]=[\Gamma][X]
$$

where $[X]$ is the matrix of eigenvectors, $[\Gamma]$ is the matrix of shape vectors and $[x]$ is the matrix of generaized eigenvectors. This approximation for $[X]$ is substituted for $[\Delta]$ in the global eigenvalue problem, equation (1), to form a reduced eigenvalue problem

$$
[\tilde{K}][\mathrm{X}]=\Omega^{2}[\tilde{\mathrm{M}}][\mathrm{X}]
$$

which can be solved inexpensively for the generalized eigenvectors $[x]$. The eigenvectors, $[X]$, which are an approximate solution to the global eigenproblem can be computed from equation (A1). To refine this approximate solution, simultaneous inverse iteration is performed on the global eigenvalue problem using $[X]$, and a new matrix $[\mathrm{r}]$ is produced. Equation (A2) can then be solved for a new, more accurate set of generalized eigenvectors $[x]$. The process is repeated until the required accuracy is attained; this constitutes the refined analysis.

It can be seen that the operations indicated by equations (A1) and (A2) are identical to the operations in the approximate vibration analysis (equations 2 and 6). Thus, the approximate vibration analysis is equivalent to one step in the subspace iteration method without the operation of simultaneous inverse 
iteration (i.e., calculating a new matrix $[\Gamma]$ ).

In both the approximate vibration analysis and the refined, global vibration analysis, the eigenvectors, $[X]$, can be calculated accurately from equation (A1) when $[\ulcorner]$ is a close approximation to $[X]$. This is the case when the structure is modified only slightly from the point where the previous refined vibration analysis was performed. At the beginning of a cycle (other than the first) in the design process, the global finite element vibration problem for the modified structure is solved by the subspace iteration method previously described. However, an excellent matrix of shape vectors $[\Gamma]$ is available from the previously calculated eigenvectors. Using the old eigenvectors as initial approximations, it typically requires only 2 or 3 iterations of the subspace technique to calculate the new modes to the required accuracy compared with 10 to 15 iterations initially. Thus, each new solution of the global vibration problem is performed for significantly less cost than the initial solution. 


\section{APPENDIX B}

\section{COMPUTER IMPLEMENTATION OF THE RESIZING PROCEDURE}

The resizing procedure is implemented within the EAL (Engineering Analysis Language) system (ref. 11) which is a derivative of the SPAR structural analys is system (ref. 12). In addition, computational modules from the PARS flutter resizing procedure (refs. 3 and 13) and the general purpose optimizer, CONMIN, (ref. 7) were added to the EAL system. The flexible design of EAL, which includes extensive facilities for data management and procedure control, allows implementation of general engineering computational tasks. This can be done by using available computational modules within EAL or by adding additional modules to be executed under EAL control. Both features were utilized in designing the flutter resizing procedure used in this study.

The EAL system and its predecessor, SPAR, are composed of a number of essentially independent computational modules (called processors) which communicate with each other through a global data base. Any block of data can be accessed by name from any processor in the system. Some processors perform fairly specific tasks such as factoring a global stiffness matrix; however, other processors are designed to perform such general tasks as adding two matrices or printing a particular block of data. The user has a considerable amount of control over both classes of processors by determining the order of their execution and the particular data operated on by them. This allows sequences of EAL commands to be written to perform complicated engineering tasks.

PARS processors for design variable definition, calculation of uns teady aerodynamic loads, solution of the reduced vibration problem, and calculation of the continuous flutter constraints and their derivatives were added to EAL. The addition of the PARS computational modules to EAL was fairly straight forward since the PARS modules were originally designed as SPAR compatible processors. That is, they were designed to retrieve and store data in the SPAR data base. A11 data transfer between original EAL processors and the new processors from PARS is through the data base. Any incompatibilities between the data requirements of the different processors were solved with simple utilities provided in 
EAL for reformatting of data blocks.

The advantage of imbedding the PARS processors and the CONMIN optimizer in EAL is that it allows use of all EAL capabilities for control of the procedure flow. EAL colmunands are provided for looping through sequences of commands, controlled branching in the command stream and the execution of independent blocks of commands which are similar to FORTRAN subroutines. The approximate analys is loop and the strategy for vibration mode updating are easily implemented using EAL control commands.

Having the control commands and the data management facilities available to the user makes it an easy task not just to design and implement a particular engineering analysis but to quickly modify it. This allowed considerable experimentation in the flutter resizing procedure without making any changes to the lower-level FORTRAN code in the processors themselves. 
REFERENCES

1. Stroud, W. Jefferson: Automated Structural Design with Aeroelastic Constraints: A Review and Assessment of the State of the Art. Presented at the ASME Symposium on Structural Optimization, L. A. Schmit, Jr., ed. AMD, Vol. 7, ASME, 1974, pp. 77-118.

2. McCullers, L. A. and Lynch, R. W.: Dynamic Characteristics of Advanced Filamentary Composite Structures, Volume II - Aeroelastic Synthesis Procedure Development. AFFDL-TR-73-111, Volume II, September 1974.

3. Haftka, R. T. and Prasad, B.: Programs for Analysis and Resizing of Complex Structures. Presented at Symposium on Future Trends in Computerized Structural Analysis and Synthesis, Washington, DC, October 30November 1, 1978. Proceedings entitled "Trends in Computerized Structural Analys is and Synthesis," Pergamon Press, New York, 1978, pp. $323-330$.

4. Cooper, Paul A. and Heldenfels, Richard R.: The NASA Structures and Materials Research Program for Supersonic Cruise Aircraft. Astronautics and Aeronautics, May 1976, pp. 26-37.

5. Sobieszczanski-Sobieski, J.; Gross, D.; Kurtze, W.; Newsom, J.; Wrenn, G.; and Greene, W.: Supersonic Cruise Research Aircraft Structural Studies: Methods and Results. 1979 Supersonic Cruise Research Conference, NASA LaRC, Hampton, Virginia, November 1979, NASA CP-2108, pp. 617-656.

6. Sobieszczanski, J.: Sizing of Complex Structures by the Integration of Several Optimal Design Algorithms. AGARD Lecture Series No. 70 on Structural Optimization, AGARD-LS-70, September 1974.

7. Vanderplaats, G. N.: CONMIN - A Fortran Program for Constrained Function Minimization, Users Manual. NASA TM X-62282, 1973. 
8. Stroud, W. Jefferson; Agranoff, Nancy; and Anderson, Melvin S.: MinimumMass Design of Filamentary Composite Panels Under Combined Loads: Design Procedure Based on a Rigorous Buckling Analysis. NASA TN D-8417, JuTy 1977.

9. Desmarais, Robert N.; and Bennett, Robert M.: User's Guide for a Modular Flutter Analys is Software System (FAST Version 1.0); NASA TM-78720, May 1978.

10. Bathe, J. and Wilson, E. L.: Numerical Methods in Finite Element Analysis. Prentice Hall, 1976.

11. Whetstone, W. D.: Engineering Data Management and Structure of Program Functions in New Techniques in Structural Analysis by Compute (Compiled by R. J. Helosh and M. Salana) ASCE Preprint 3601, ASCE Convention and Exposition, Boston, Mass., 1979.

12. Whetstone, W. D.: SPAR Structural Ana Tys is System Reference Manual System Leve1 13A, Volume I - Program Execution, NASA CR-158970-1, 1978.

13. Haftka, R. T.; Prasad, B.; and Tsach, U.: PARS - Programs for Analysis and Resizing of Structures - User Manual. NASA CR-159007, Apri1 1979. 
TABLE I. - COMPARISON BETWEEN THE LOWEST ELASTIC NATURAL FREQUENCIES ( $\mathrm{HZ}$ ) OF THE STRENGTH-SIZED AND FLUTTER-RESIZED (CASE D) AIRCRAFT

\begin{tabular}{|c|c|c|}
\hline Strength Sized & Case D & \% Change \\
\hline 1.136 & 1.148 & 1.0 \\
1.372 & 1.378 & .4 \\
2.146 & 2.269 & 5.4 \\
2.723 & 2.780 & 2.1 \\
3.272 & 3.460 & 5.4 \\
3.813 & 4.973 & 4.0 \\
4.453 & 4.997 & 10.9 \\
5.248 & 5.276 & .5 \\
5.624 & 5.652 & .5 \\
\hline
\end{tabular}




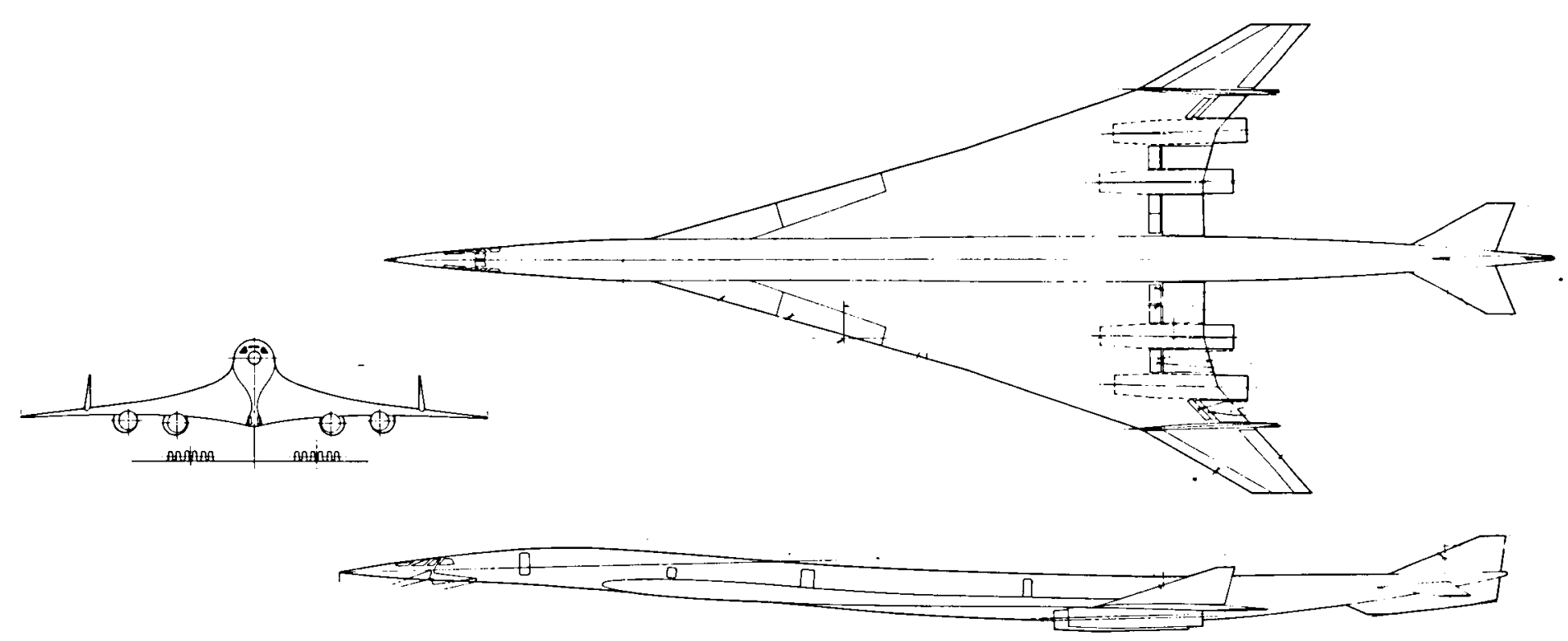

TAKE-OFF GROSS WEIGHT $311163 \mathrm{~kg}(686000 \mathrm{lb})$ OPERATION EMPTY WEIGHT $130618 \mathrm{~kg}(287965 \mathrm{lb})$ PAYLOAD LENGTH $25880 \mathrm{~kg} \quad(57057 \mathrm{lb})$

SPAN CRUISE SPEED

$93 \mathrm{~m}(305 \mathrm{ft})$

$39 \mathrm{~m} \quad(127 \mathrm{ft})$

$M=2.7$

Figure 1.- Basic characteristics of the arrow wing supersonic cruise vehicle. 


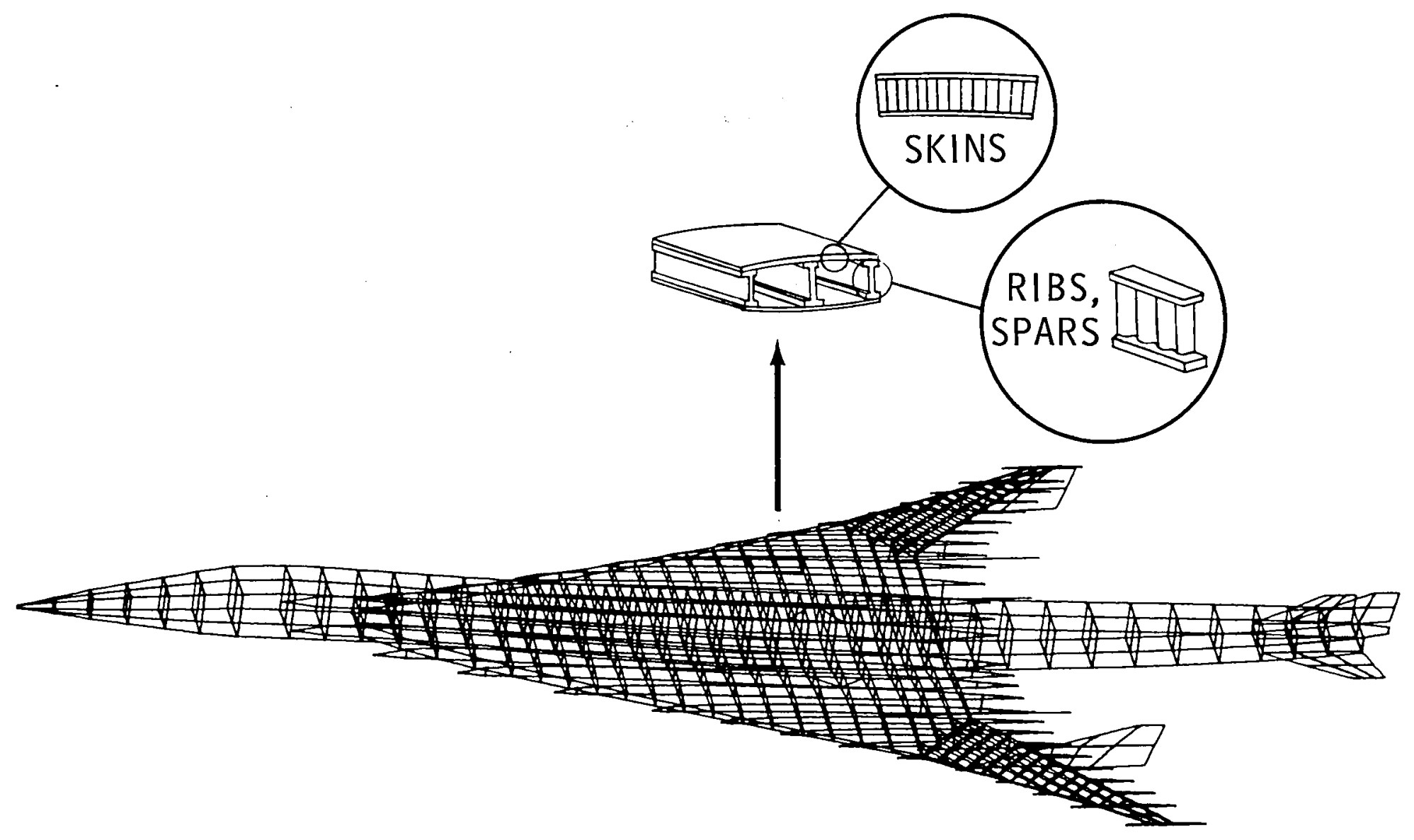



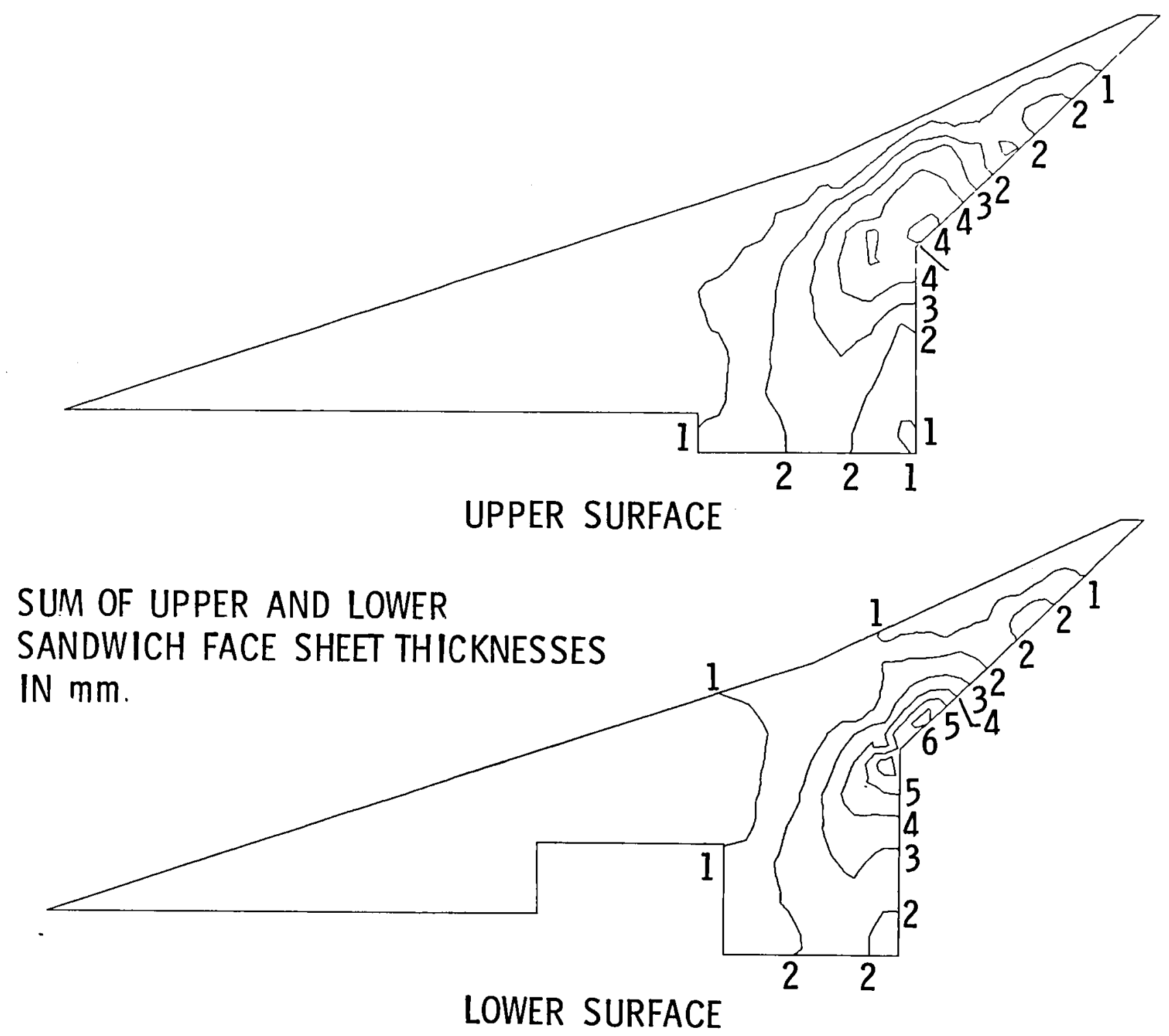

Figure 3.- Skin thickness contours of the wing cover panels for the strength-sized airframe. 

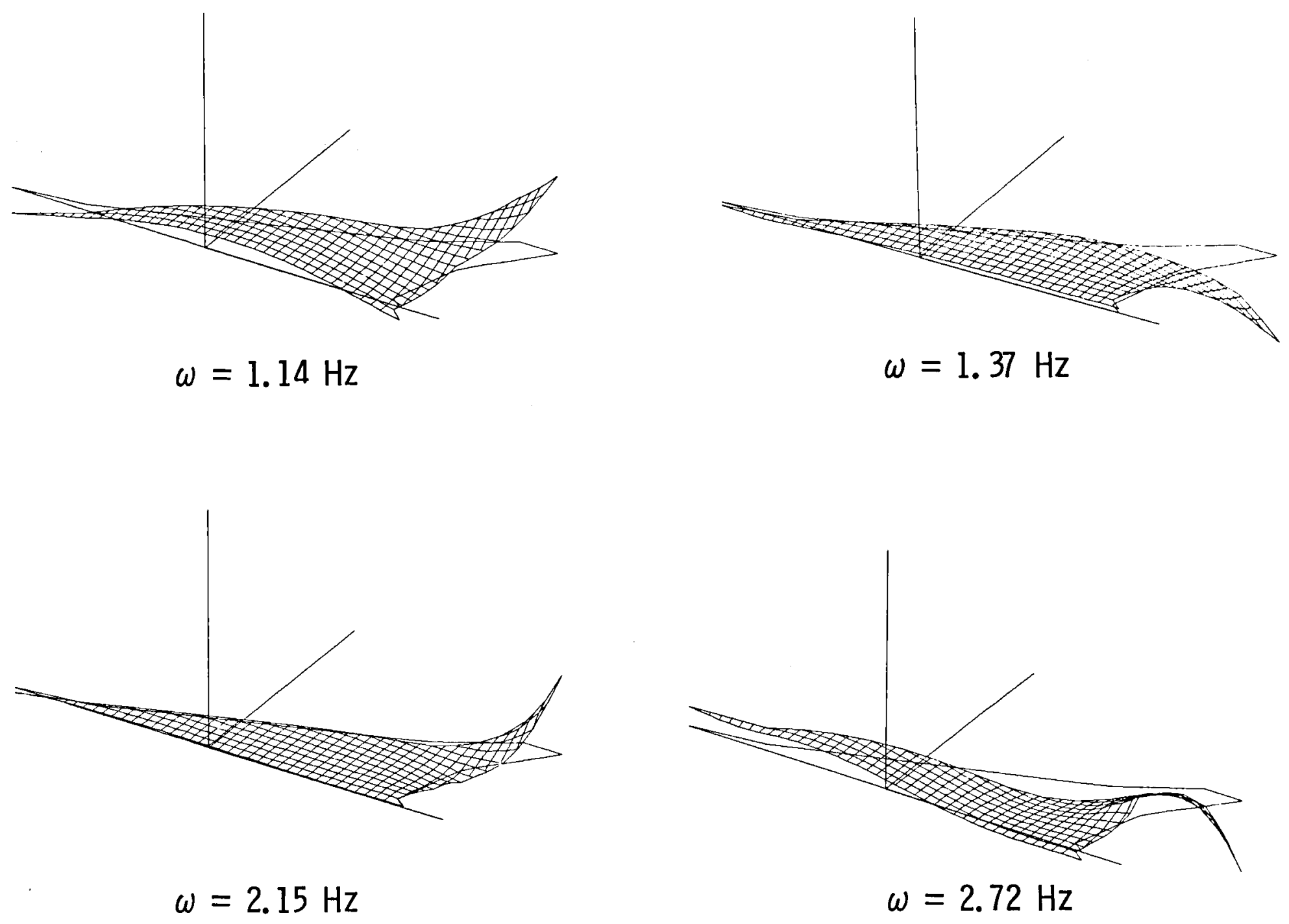

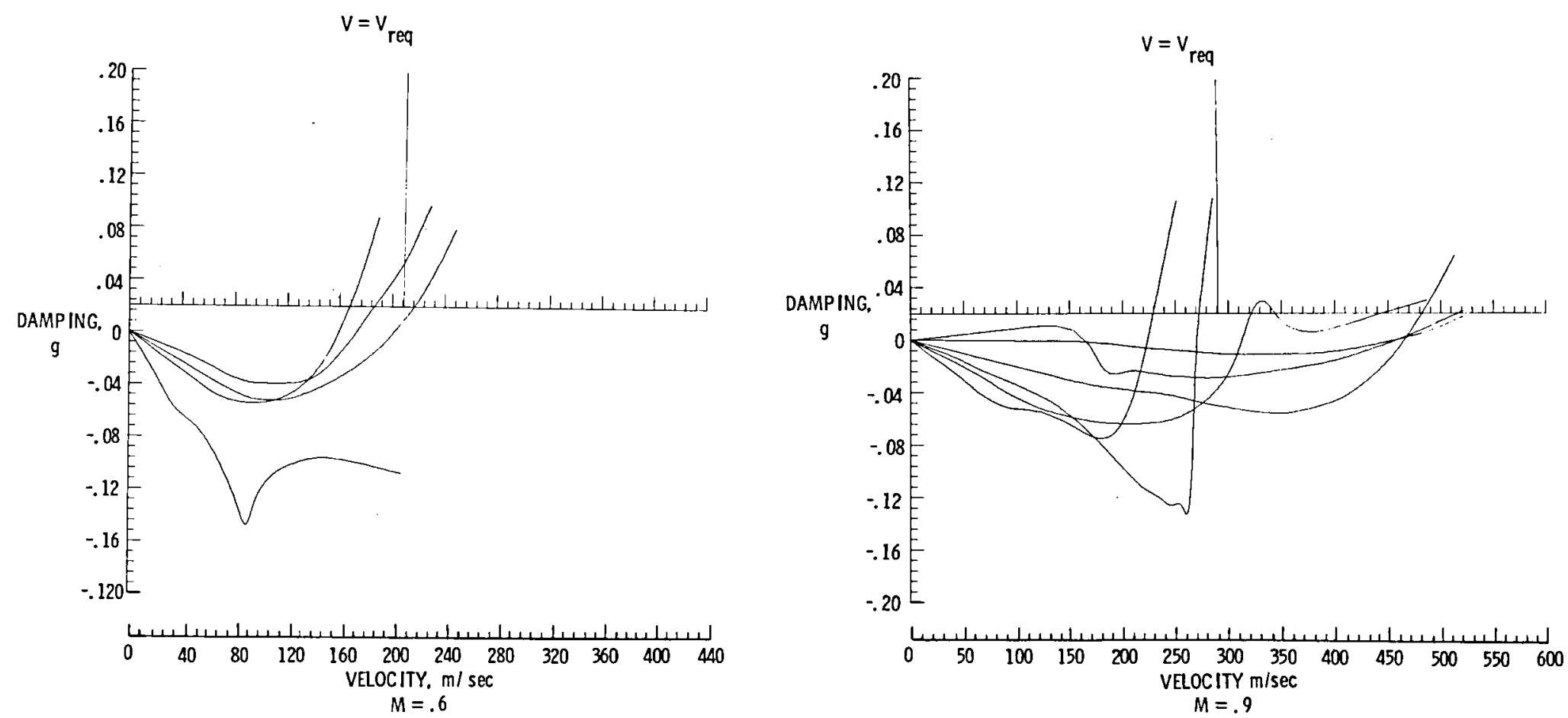

Figure 5.- V-g plots for the strength-sized aircraft. 


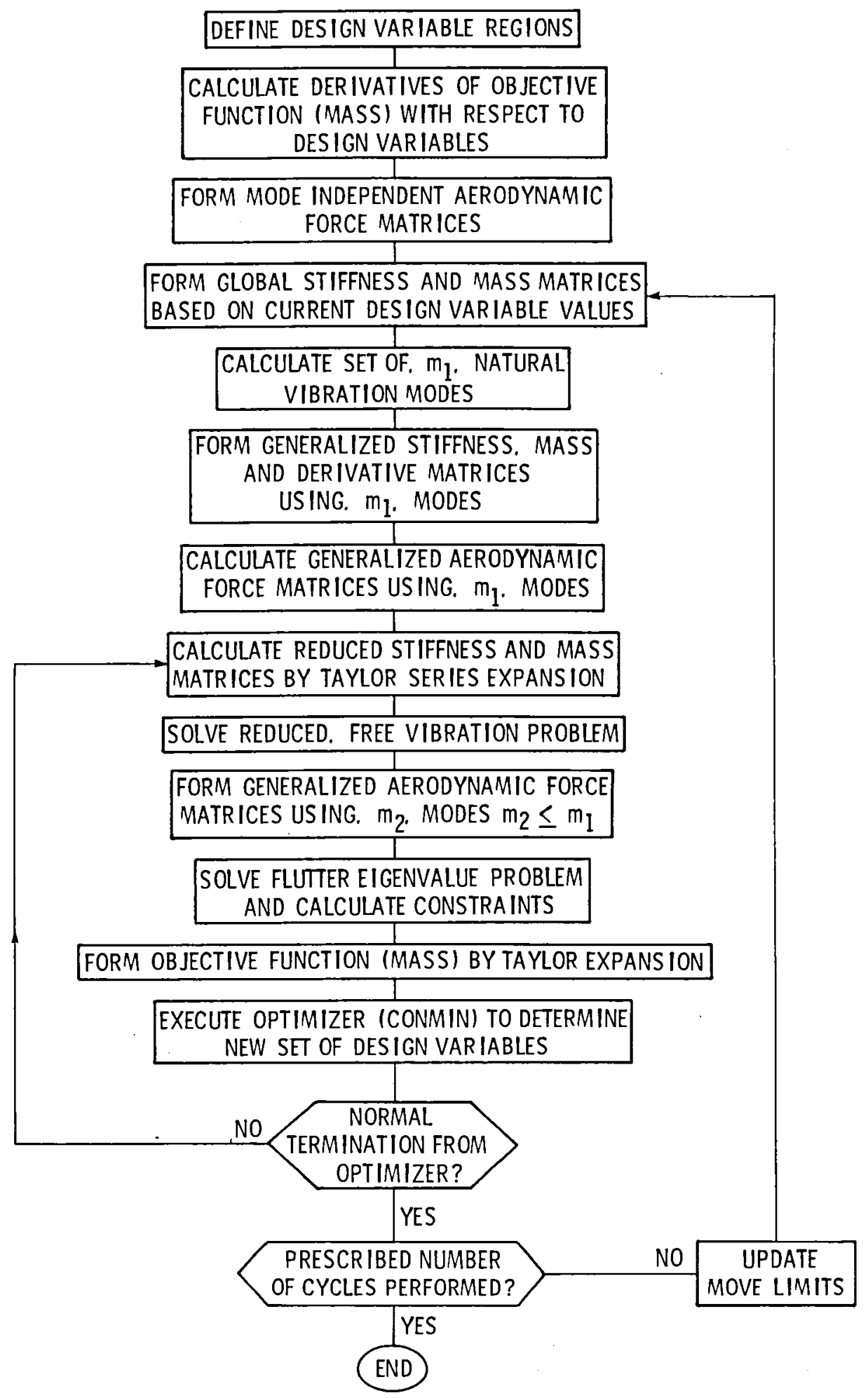

Figure 6. - Flow chart of the resizing procedure. 


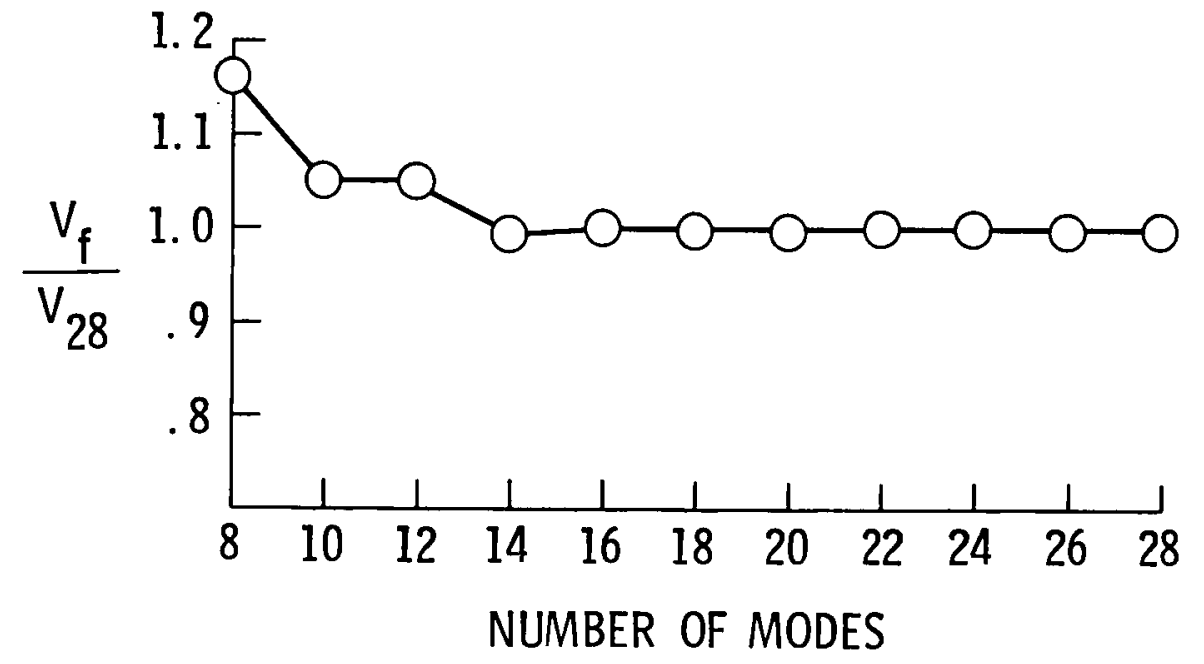

$M=.6$

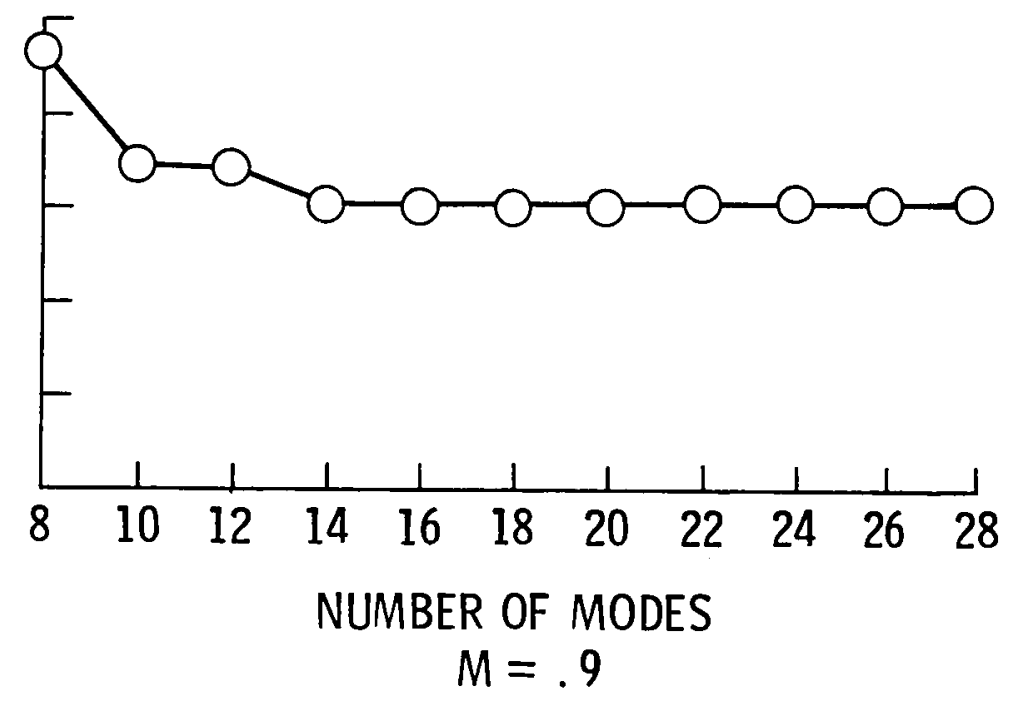

Figure 7.- Convergence study on the number of natural vibration modes used in the flutter eigenvalue analysis. 


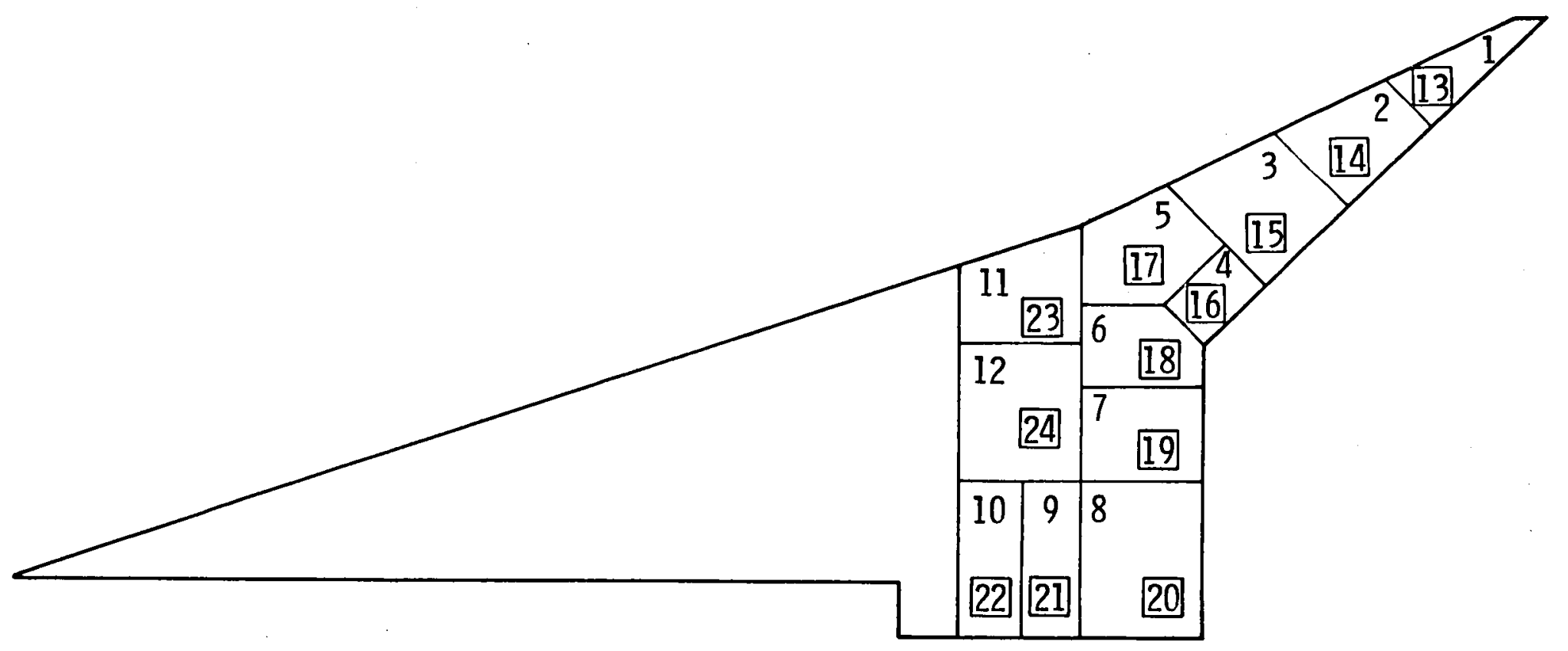

$\square$ INDICATES A DESIGN VAR IABLE REGION ON THE WING LOWER SURFACE FOR DESIGN VARIABLE SET $\square$.

Figure 8.- Cover panel thickness regions controlled by design variables. 


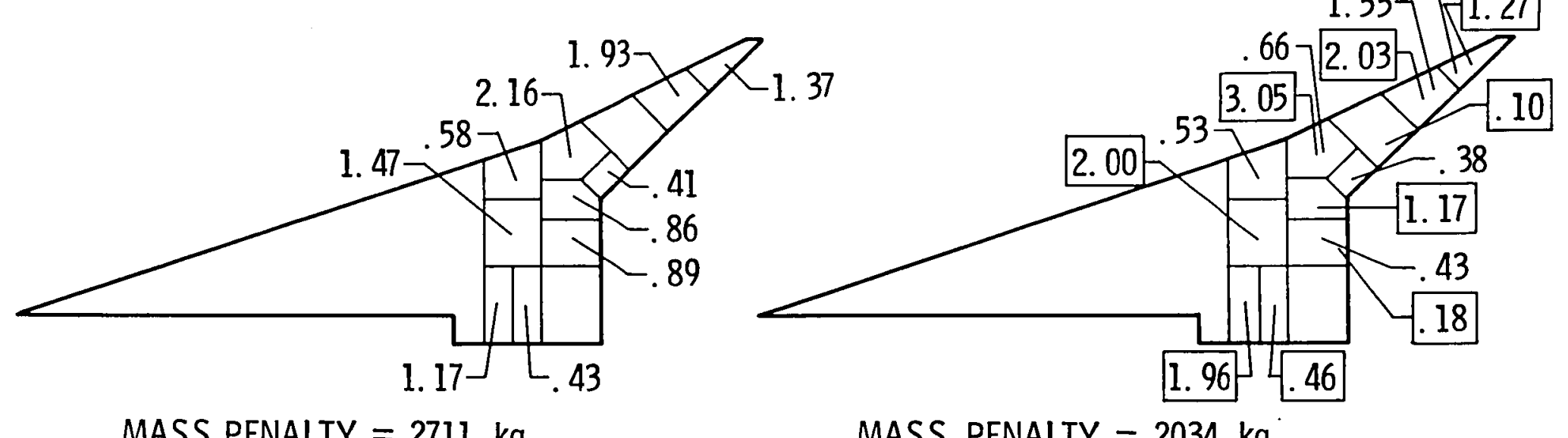

MASS PENALTY $=2711 \mathrm{~kg}$

(a) CASE A

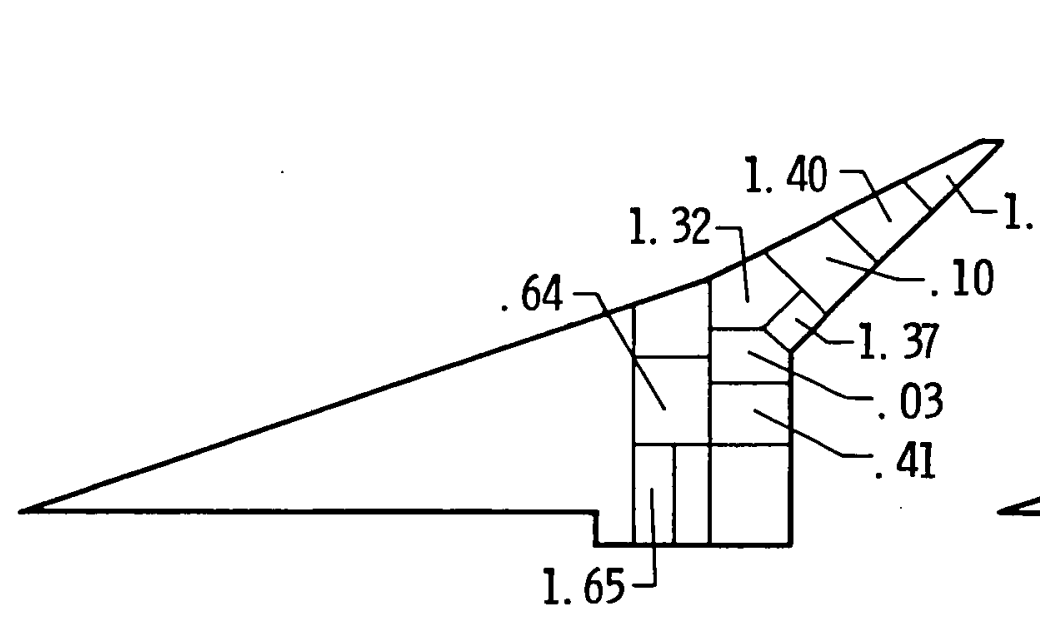

MASS. PENALTY $=1822 \mathrm{~kg}$

(c) CASE C
MASS PENALTY $=2034 \mathrm{~kg}$

(b) CASE B

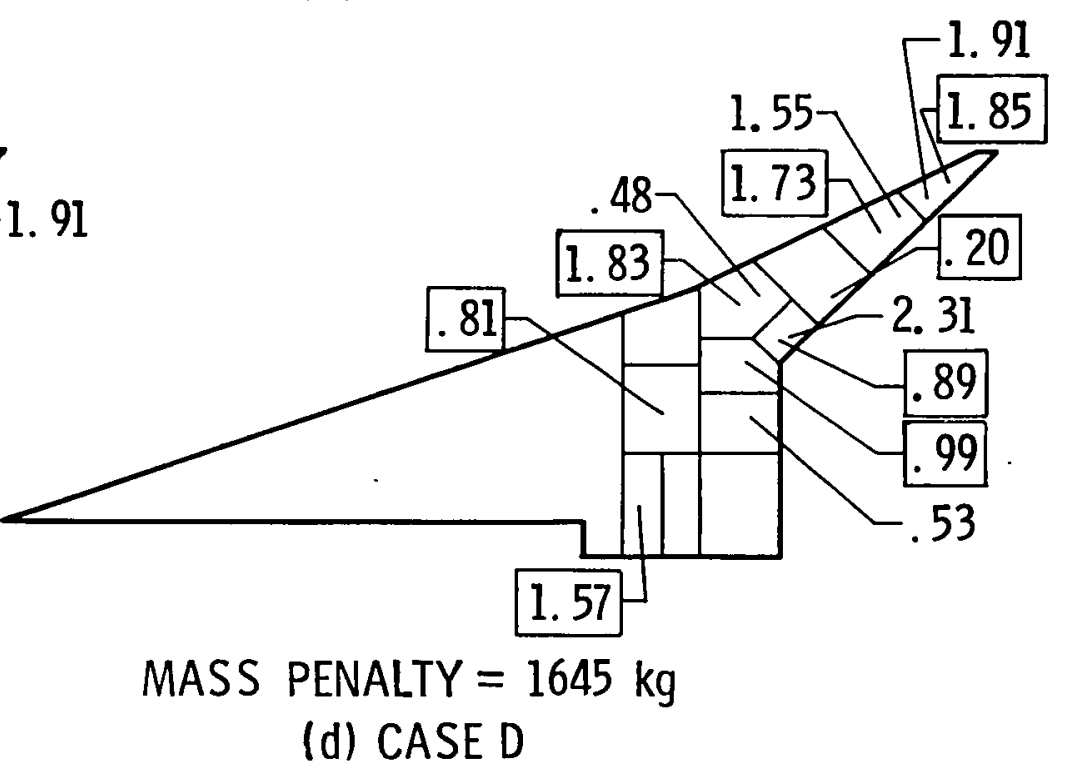

Figure 9.- Final design variable values for the four cases. Values are sums of upper and lower sandwich face sheet thickness additions in $\mathrm{mm}$. 


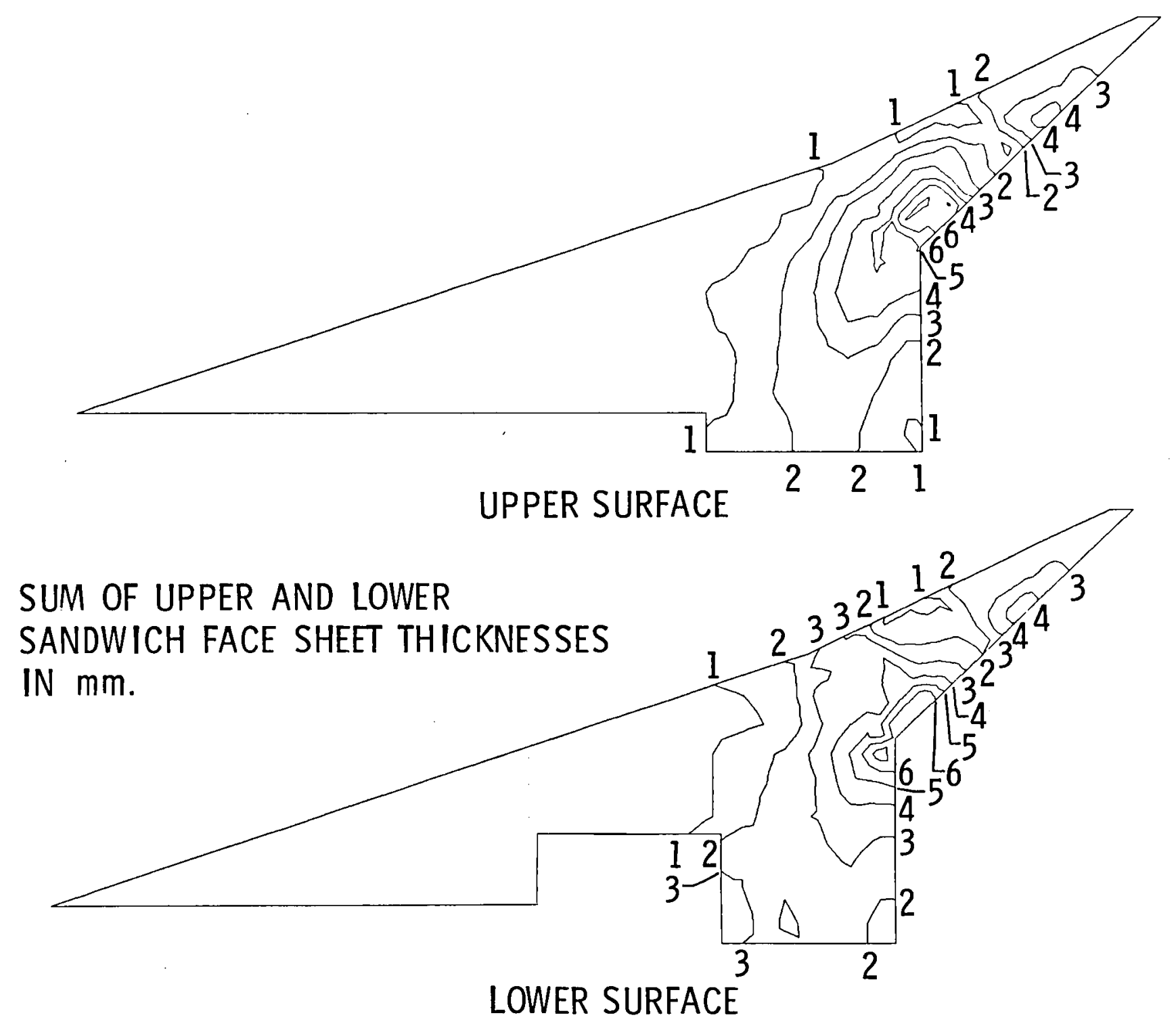
meet flutter requirements (case D). 

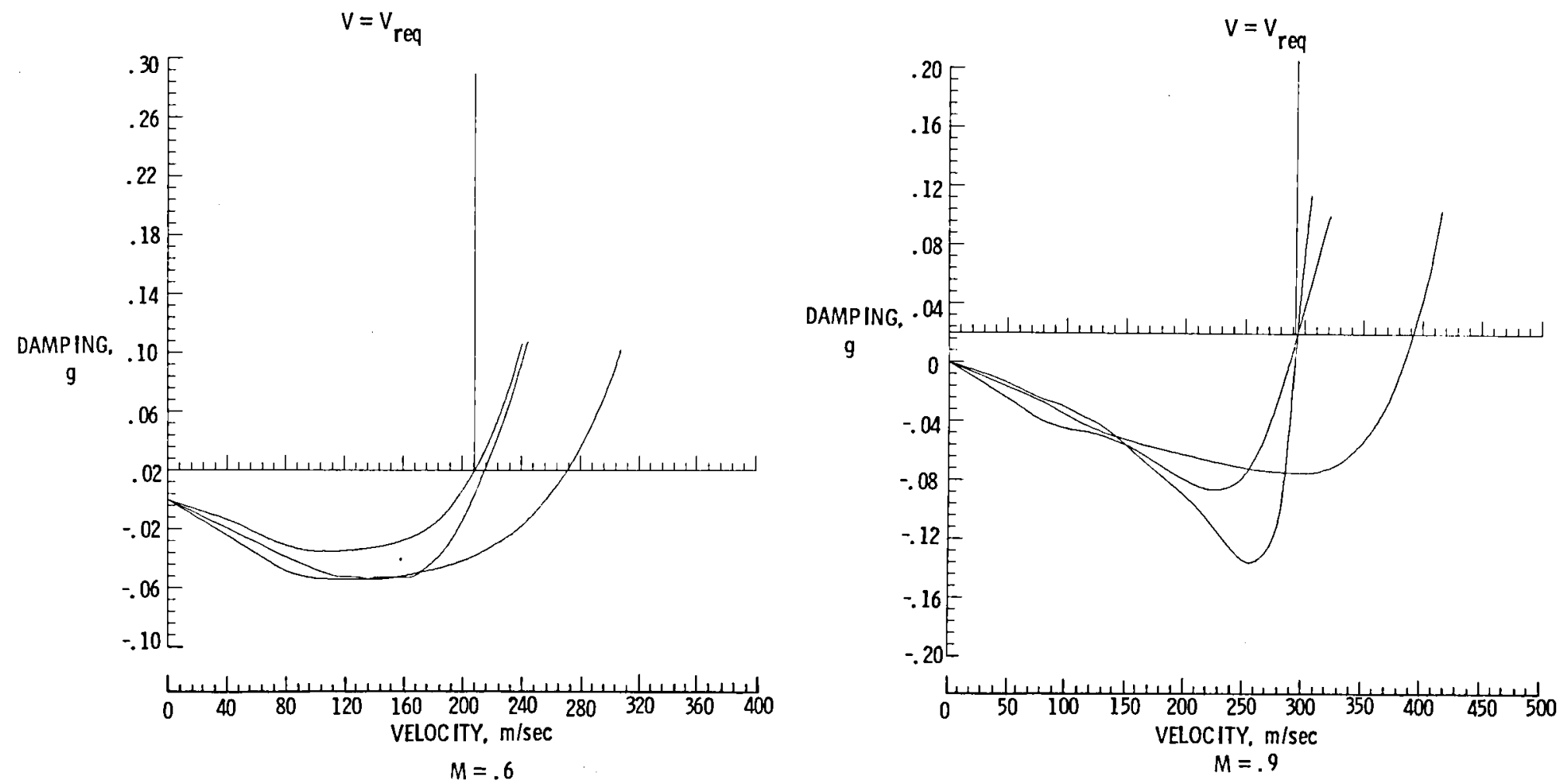

Figure 11.- Vag plots for the aircraft resized to meet flutter requirements (case D). 

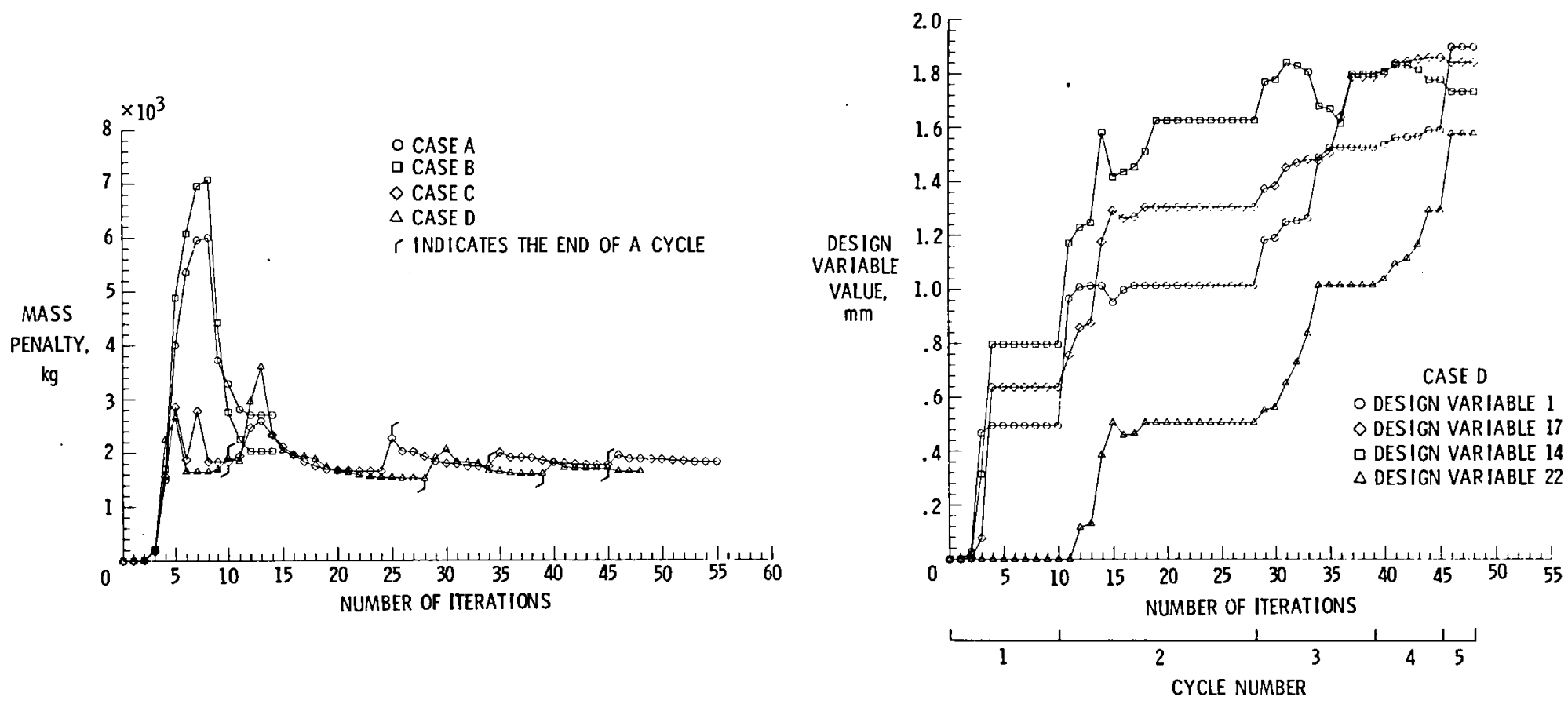

$\omega$

Figure 12,- Design histories of mass penalties and selected design variable values. 
$\cdot$ 


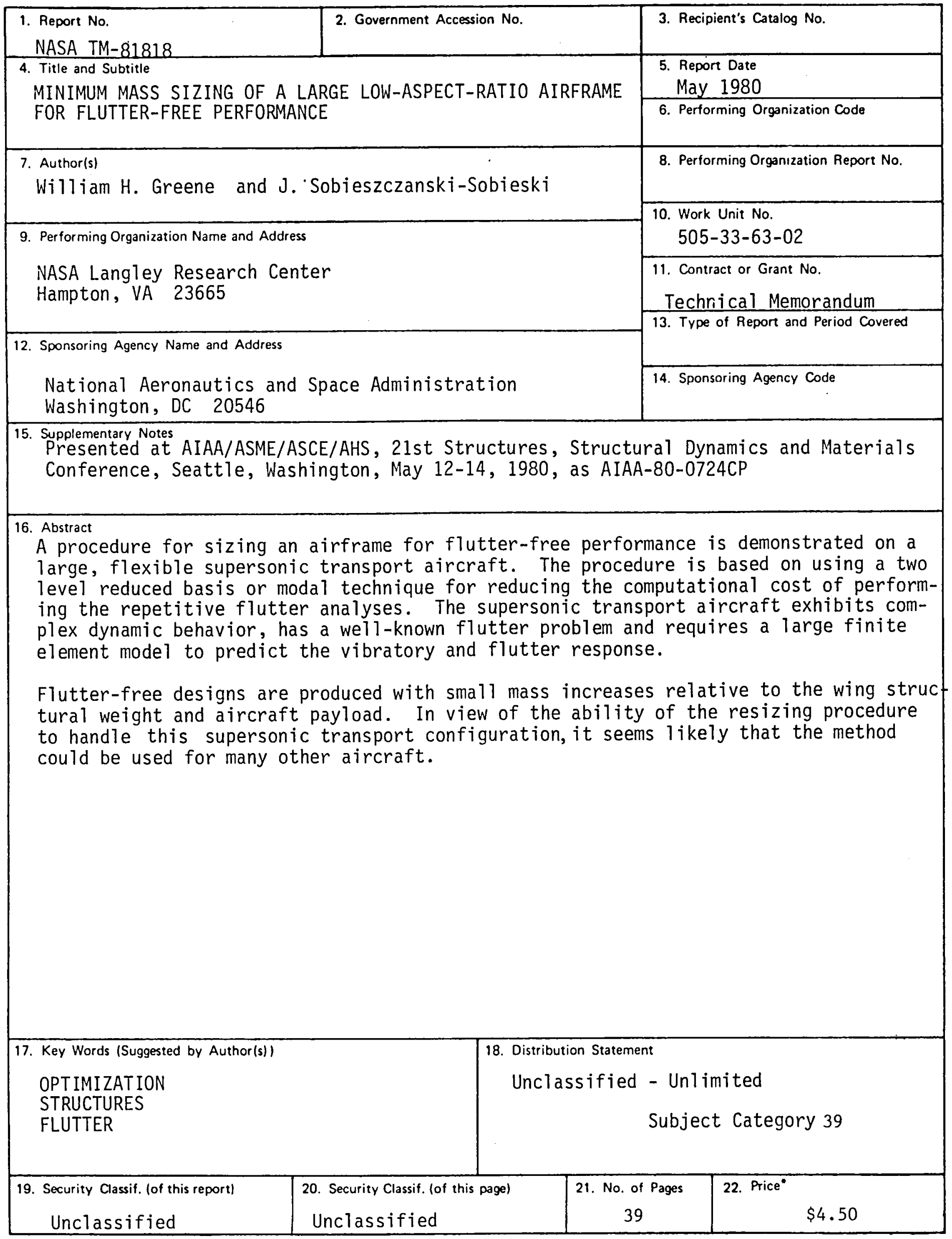

* For sale by the National Technical Information Service, Springfield, Virginia 22161 

3
$?$
1
1
1

1 